\title{
Efficiency comparison between dry and humid cultures for clinical outcome of the in vitro fertilization-embryo transfer
}

\section{Weihai Xu ( $\nabla$ xuweihai@hmc.edu.cn )}

Zhejiang Provincial People's Hospital, Hangzhou Medical College

\section{Lin Zhang}

Zhejiang Provincial People's Hospital, Hangzhou Medical College

\section{Ling Zhang}

Zhejiang Provincial People's Hospital, Hangzhou Medical College

\section{Shishi Li}

Zhejiang Provincial People's Hospital, Hangzhou Medical university

\section{Jing Shu}

Zhejiang Provincial People's Hospital, Hangzhou Medical College

\section{Research}

Keywords: Embryo, culture, In-vitro fertilization, embryo transfer, osmotic pressure

Posted Date: March 30th, 2021

DOI: https://doi.org/10.21203/rs.3.rs-335124/v1

License: (c) (1) This work is licensed under a Creative Commons Attribution 4.0 International License. Read Full License 


\section{Abstract}

Background: In this study, we compared the in vitro embryo development, embryo transfer outcome and the offspring outcome in the in vitro fertilization-embryo transfer (IVF-ET) between dry culture (DC) and humid culture $(\mathrm{HC})$.

Methods: Our study was divided into two parts. Firstly, we determined the fertilization rate, cleavage rate and high-quality embryo rate from 21 cycles in the DC group ( $\mathrm{N}=262$ oocytes) and HC group ( $\mathrm{N}=263$ oocytes). Secondly, we determined the embryo transfer outcome and the offspring outcome in DC group ( $\mathrm{N}=184$ cycles) and $\mathrm{HC}$ group ( $\mathrm{N}=136$ cycles).

Results: Compared with the $\mathrm{HC}$ group, significant increase was observed in the high-quality embryo rate $(66.1 .2 \%$ vs. $55.3 \%, p=0.037)$ and implantation rate $(49.8 \%$ vs. $40.6 \%, p=0.027)$ in the $\mathrm{DC}$ group. No statistical differences were observed in the pregnant outcome and birth defect of the offspring $(p>0.05)$. Compared with HC, DC was associated with a higher high-quality embryo rate and a higher implantation rate after embryo transfer.

Conclusions: No statistical differences were noticed in the offspring conditions between the two culture modes. Taken together, DC may serve as a promising method for IVF-ET.

\section{Background}

In vitro fertilization-embryo transfer (IVF-ET) is considered a promising treatment option for the infertile couples. Its core element is to achieve IVF and select embryos with developmental potency for the transfer and/or cryopreservation. For a long time, humid culture (HC) has been conventionally utilized for the in vitro embryo culture. It provides a saturated humidity in the culture environment for embryo by means of water vaporization in an active manner or humidification based on disposable humidification flask. On this basis, the osmotic pressure of culture medium is stable [1, 2], contributing to the culture of zygote and embryo. Therefore, it is recommended as the major method for embryo culture [3].

As a new technique, dry culture (DC) has been used in the human embryo culture. Unlike the HC inducing generation of microorganisms that may affect the embryonic growth, DC involving a relatively dry environment can reduce the risks of microorganism growth [4]. In addition, by integrating with the TimeLapse Imagine (TLI) technique, DC promotes the comprehensive monitoring of the embryonic growth.

To date, few studies have been carried out to investigate the efficiency of DC in clinical settings $[5,6]$. In a previous study, Fawzy et al indicated that the developed high-quality embryo rate on day 3 was significantly lower in the DC group than in the HC group ( $65 \%$ vs. $83 \%, p<0.0001)$ [7]. In contrast, Elqusi et al reported that the high-quality blastocyst rate in the DC group was significantly higher than that of the HC group (62.9\% vs. 43.5\%) [8]. Furthermore, little is known about the effects of DC on the outcome of embryo transfer and offspring. In this study, we compared the effects of DC and $\mathrm{HC}$ on the offspring and evaluated the feasibility of the IVF-ET on the DC and HC. 


\section{Methods}

\section{Devices}

The EC6-230LE dry incubator (Astec, Japan) and APM-50 D humid incubator (Astec, Japan) were used in this study. The temperature, $\mathrm{CO}_{2}$ and $\mathrm{O}_{2}$ concentration were determined per day before the experiments.

\section{Subjects}

The IVF-ET related data were collected from 501 subjects presenting to the Reproductive Medicine Center, Zhejiang Provincial People's Hospital between November 2016 and October 2017. The inclusion criteria were as follows: (i) female aged $\leq 38 \mathrm{yrs}$; (ii) those received frozen-thawed embryo transfer (FET) as the first embryo transfer; (iii) those received transfer of D3 high-quality embryos. Those with the following conditions were excluded from this study: (i) any individual of the couples presenting chromosomal anomaly; (ii) those with rescue intracytoplasmic sperm injection (ICSI) cycles after the IVF.

\section{Experimental design}

Twenty-one cycles with oocytes retrieval of $\geq 20$ was collected in this study. The oocytes in each cycle were randomly divided into DC group ( $\mathrm{N}=262$ oocytes) and HC group ( $\mathrm{N}=263$ oocytes). Then we determined the in vitro culture efficiency including the fertilization rate, cleavage rate and high-quality embryo rate. In order to eliminate the effects of other factors on the clinical outcome, we analyzed the clinical outcome of the embryo and offspring conditions based on different culture model. Eventually, 352 cycles met the inclusion criteria, and 320 cycles were finally included in this study, consisting of DC group ( $\mathrm{N}=184)$ and $\mathrm{HC}$ group $(\mathrm{N}=136)$, after excluding 5 with female chromosome abnormality, 5 with male chromosome abnormality, and 22 with rescue ICSI.

\section{IVF therapy}

The oocytes retrieval, IVF and embryo culture were conducted in our lab using the GnRH antagonist protocol, long protocol, and minimal stimulation protocol according to the previous description $[9,10]$. The follicle-stimulating hormone (FSH), human menopause gonadotropin (HMG) and clomiphene were utilized to stimulate the follicular development according to the ovary reserve and response. In cases of a diameter of $>17 \mathrm{~mm}$ in two follicles, or a diameter of $>18 \mathrm{~mm}$ in one follicle, human chorionic gonadotrophin (HCG) and/or GnRH-a were administrated, followed by oocytes retrieval within 36-38 hrs.

In order to avoid the influences of repetitive door open during the culture procedure, the chamber was performed independently for the in vitro culture cycle. The COOK sequential media (Cook Medical, Brisbane, Australia) was utilized for the IVF and embryo culture. The droplets prepared based on fertilization medium $(\mathrm{FM}, 80 \mu \mathrm{l})$ and cleavage medium $(\mathrm{CM}, 30 \mu \mathrm{l})$ were transferred to petri dishes $(60 \times 15$, 353652, Falcon), followed by covering with $8.5 \mathrm{ml}$ embryo culture oil (ART-4008-5P, Origio). Upon oocytes retrieval, conventional IVF or ICSI was performed. On day 3 , the high-quality embryo was selected according to the ASBIR criteria [11] for the subsequent embryo cryopreservation. 
The endometrial preparation protocol of the FET cycle consists of natural cycle, hormone replacement therapy protocol and ovarian stimulation protocol. Based on the endometrium development conditions, one or two D3 high-quality frozen embryo was thawed, followed by transfer. Then the serum HCG was measured on day 12 to determine whether the female was pregnant. The clinical pregnancy was defined as presence of gestational sac by B-ultrasonography on day 35 after transfer. Those presenting no fetal heart or loss of fetal heart at week 12 were defined as early abortion. Ongoing pregnancy was defined as development of gestational sac for at least 12 weeks.

\section{Statistical analysis}

SPSS 19.0 software was used for the data analysis. Measurement data that were normally distributed were presented as mean \pm standard deviation. Inter-group comparison was conducted using Student's ttest. The comparison of numeration data was conducted using the Chi square test. Multi-variate logistic regression analysis was used to analyze the effects of female age, fertilization protocol, number of transferred embryos, and the culture mode on the pregnancy. $p<0.05$ was statistically significant.

\section{Results}

\section{Comparison of fertilization rate, cleavage rate and high-quality embryo rate in sibling oocyte}

The fertilization rate in the DC group showed 10 no statistical difference compared with the HC group $(69.8 \%$ vs. $68.8 \%, p>0.05$, Table 1$)$. Meanwhile, there were no differences in the rate of cleavage between the two groups $(98.4 \%$ vs. $98.9 \%, p>0.05)$. The high-quality embryo rate in the DC group was significantly higher than that of the HC group ( $66.1 \%$ vs. $55.3 \%, p=0.036)$.

\section{Clinical outcome for the embryo transfer}

Compared with the DC group, there were no statistical differences in the age of the couple, infertility duration, previous history of pregnancy, causes of infertility, BMI, ovarian stimulation protocol, fertilization protocol and endometrial preparation protocol in the $\mathrm{HC}$ group $(p>0.05$, Table 2$)$. The number of transferred embryo in the DC group was significantly lower than that of the HC group $(1.73 \pm 0.44$ vs. $1.87 \pm 0.34, p=0.004)$. The implantation rate of embryo in the $D C$ group was significantly higher than that of the $\mathrm{HC}$ group $(49.8 \%$ vs. $40.6 \%, p=0.027)$. After adjusting the female age, endometrial preparation protocol, fertilization protocol and number of transferred embryos, there was no correlation between type of culture mode and the clinical outcome among cases received different transfer cycles $(\mathrm{OR}=1.339 ; 95 \%$ Cl: 0.842-2.128; $p=0.217)$.

\section{Comparison of the offspring}

In this section, we compared the offspring from the couples underwent DC and $\mathrm{HC}$. There were no statistical differences in the gestational weeks ( $35.9 \pm 2.2$ weeks vs. $36.3 \pm 1.6$ weeks, $p>0.05)$ and rate of preterm birth $(57.5 \%$ vs. $45.0 \%, p>0.05)$ of the women with Singleton or twin pregnancy between the two 
groups ( $p>0.05)$. In addition, the body weight and malformation rate also showed no statistical differences $(p>0.05$, Table 3$)$.

\section{Discussion}

This study was divided into two sections. In the first section, we determined the fertilization rate, cleavage rate and high-quality embryo rate of the 525 oocytes from 21 cycles in the DC group ( $N=262)$ and $\mathrm{HC}$ group ( $\mathrm{N}=263)$. In the second section, we determined the embryo transfer outcome and the offspring outcome from the 320 oocyte retrieval cycles in $\mathrm{DC}$ group $(\mathrm{N}=184)$ and $\mathrm{HC}$ group $(\mathrm{N}=136)$. Our data showed that there were no statistical differences in the fertilization rate and cleavage rate between the DC group and $\mathrm{HC}$ group. However, the rate of high-quality embryo in the DC group was significantly higher than that of the $\mathrm{HC}$ group. Our data showed that the embryo obtained from DC presented a higher implantation rate. Additionally, the physiological indices of the offspring delivered based on DC were similar with those based on the HC. Thus, we speculated that DC showed superiority and comparable safety in the IVF-ET compared with that of the HC.

Currently, the TLI system frequently utilized for the assisted reproductive therapy is mainly based on DC, in order to obtain high-quality embryo. In a previous study, the proportion of high-grade embryos on day 2 was significantly higher in the TLI group compared with the G185 group ( $40.4 \%$ vs. $35.2 \%$ ), while the proportion on day 2 in the TLI group was higher than that of the 8-cell stage (34.3\% vs. 25.3\%) [12]. In addition, Sciorio et al indicated that the proportion of high quality embryos was higher in the Embryoscope time-lapse incubator group compared to the benchtop incubator on day 3 (75.1 vs. $56.0 \%$, $p=0.006$ ) [13]. In a previous study, based on the in vitro culture of 571 oocytes, Elqusi et al reported that the rate of blastocyst obtained in the DC model was significantly higher than that in the HC model $(62.9 \%$ vs. $43.5 \%$ ) [8]. It implied that the DC may serve as a promising culture model for embryo for the IVF-ET.

Indeed, some studies indicated that $\mathrm{HC}$ was superior to the DC in the IVF-ET. For example, the DC mode was associated with quality decline in the embryo at the cleavage stage and a lower rate of blastocyst formation compared with the $\mathrm{HC}$ mode, resulting in a decreased implantation and ongoing pregnancy rates [7]. In addition, there was strong evidence that culture conditions with high humidity atmosphere promoted embryo development and reproductive outcome [14]. In the Cairo consensus guidelines on IVF culture conditions, the $\mathrm{DC}$ and $\mathrm{HC}$ contributed to satisfactory outcome in clinical settings, but the $\mathrm{HC}$ was proved to be more suitable for IVF-ET [3]. This is related to the fact that the humidity in the chamber can be modulated through the water evaporation in the humid incubator, which can attenuate the water loss in the culture. This avoids the potential damages to the embryonic development caused by elevation of osmotic pressure.

Compared with the previous studies $[7,15]$, the major data differences in our study may be associated with the in vitro conditions. According to the previous description, the zygotes were cultured until day 5 and day 6 without replacing the culture solution. In this process, a long-time culture may lead to loss of water content, which subsequently induced elevation in the osmotic pressure of the embryo [16]. Six 
droplets $(100 \mu \mathrm{l})$ were placed in $35 \mathrm{~mm}$ Petri dish, and $3.5 \mathrm{ml}$ mineral oil was overlaid. After 2 days of DC, the osmotic pressure showed a merely elevation of $1.8 \mathrm{mOsm}[17]$. The osmotic changes in the DC condition were about 20 mOsm after 6 days of incubation [15]. This implied that the osmotic pressure of the micro-droplets in the DC system would remarkably increase with the time. In this study, the persistent utilization of droplets for three days could avoid the potential damages to the embryo induced by the osmotic pressure changes. In fact, the slight change of osmotic pressure during the embryo culture could be effectively corrected by the glycine in the culture medium[18] and the intracellular glycine [19]. In vitro, under conditions of increased osmolarity, $50 \mathrm{mM}$ glycine can be accumulated in 1-cell mice embryos, which could balance up to about $50 \mathrm{mOsm}$ of external osmotic pressure [20]. Furthermore, increasing the oil volume for the coverage of micro-droplets may affect the efficiency of DC. For instance, Swain et al [15] indicated that the elevation of the oil volume may reduce the water loss and decrease the alternation of the osmotic pressure. In this study, we did not hold a deep investigation on the osmotic pressure changes after knowing the conclusion from Mori et al [17]. In addition, we did not investigate the optimal volume of the oil for the embryo culture; however, a volume of $8.5 \mathrm{ml}$ was effective for the embryo culture. Therefore, appropriate culture method should be adopted for the DC in order to effective control the potential risks induced by changes of osmotic pressure.

Indeed, there are some limitations in our study. For instance, the sample size is not large, lack of multicentered data. In future, we will focus on data collection of the clinical treatment cycle performed in multiple centers, in order to obtain more representative data.

\section{Conclusions}

In summary, DC serving as a novel type for the embryo culture showed a higher high-quality embryo rate compared with the HC. Besides, DC was associated with a higher implantation rate after embryo transfer. There were no statistical differences in the offspring conditions between the $\mathrm{DC}$ and $\mathrm{HC}$. Taken together, the DC is a promising method for the embryo culture of IVF-ET.

\section{Declarations}

\section{Ethics approval and consent to participate}

This study was approved by the Reproductive Ethics Committee of Zhejiang Provincial People's Hospital (Protocol \# SZ2016013). Each patient signed the informed consent.

\section{Consent for publication}

Not applicable.

\section{Availability of data and materials}


All the data were available upon appropriate request. The dataset(s) supporting the conclusions of this article is(are) included within the article (and its additional file(s)).

\section{Competing interests}

The authors declare that there is no conflict of interest regarding the publication of this paper.

\section{Funding}

Not applicable.

\section{Authors' contributions}

XWH wrote the manuscript; SJ revised the manuscript; LSS and ZL did the data analysis; ZL did the data collection. The authors read and approved the final manuscript.

\section{Acknowledgements}

Not applicable.

\section{References}

1. K. Yumoto, K. Iwata, M. Sugishima, J. Yamauchi, M. Nakaoka, M. Tsuneto, T. Shimura, S. Flaherty, Y. Mio, Unstable osmolality of microdrops cultured in non-humidified incubators, J Assist Reprod Genet. 36 (2019) 1571-7. https://doi.org/10.1007/s10815-019-01515-9.

2. C. Albert, N. Gonzalez, J. Marcos, L. Alegre, B.A. Ruiz, J.M. De Los Santos, M. Meseguer, The effect of high humidity culture conditions over embryo development: a continuous embryo monitoring assessment, Reproductive Biomedicine Online. 37 (2018) e15-e6

3. C. Consensus Group, 'There is only one thing that is truly important in an IVF laboratory: everything' Cairo Consensus Guidelines on IVF Culture Conditions, Reprod Biomed Online. 40 (2020) 33-60. https://doi.org/10.1016/j.rbmo.2019.10.003.

4. R.J. Geraghty, A. Capes-Davis, J.M. Davis, J. Downward, R.I. Freshney, I. Knezevic, R. Lovell-Badge, J.R. Masters, J. Meredith, G.N. Stacey, P. Thraves, M. Vias, Guidelines for the use of cell lines in biomedical research, Br J Cancer. 111 (2014) 1021-46. https://doi.org/10.1038/bjc.2014.166.

5. J.M. Baltz, Media composition: salts and osmolality, Methods Mol Biol. 912 (2012) 61-80. https://doi.org/10.1007/978-1-61779-971-6_5.

6. S.H. McKiernan, B.D. Bavister, Environmental variables influencing in vitro development of hamster 2cell embryos to the blastocyst stage, Biol Reprod. 43 (1990) 404-13. https://doi.org/10.1095/biolreprod43.3.404.

7. M. Fawzy, M.Y. AbdelRahman, M.H. Zidan, F.F. Abdel Hafez, H. Abdelghafar, H. Al-Inany, M.A. Bedaiwy, Humid versus dry incubator: a prospective, randomized, controlled trial, Fertil Steril. 108 (2017) 277-83. https://doi.org/10.1016/j.fertnstert.2017.05.036. 
8. K.M. Elqusi, A.A. Hussin, H.A. Alkhader, H. Zaki, Humid versus dry bench-top incubator: a case control study, Fertility and Sterility. 110 (2018) e356

9. R. Orvieto, R. Nahum, E. Zohav, G. Liberty, E.Y. Anteby, S. Meltcer, GnRH-agonist ovulation trigger in patients undergoing controlled ovarian hyperstimulation for IVF with ultrashort flare $\mathrm{GnRH}$-agonist combined with multidose GnRH-antagonist protocol, Gynecol Endocrinol. 29 (2013) 51-3. https://doi.org/10.3109/09513590.2012.705376.

10. L. Rinaldi, F. Lisi, H. Selman, Mild/minimal stimulation protocol for ovarian stimulation of patients at high risk of developing ovarian hyperstimulation syndrome, J Endocrinol Invest. 37 (2014) 65-70. https://doi.org/10.1007/s40618-013-0021-1.

11. The Istanbul consensus workshop on embryo assessment: proceedings of an expert meeting, Hum Reprod. 26 (2011) 1270-83. https://doi.org/10.1093/humrep/der037.

12. J. Barberet, J. Chammas, C. Bruno, E. Valot, C. Vuillemin, L. Jonval, C. Choux, P. Sagot, A. Soudry, P. Fauque, Randomized controlled trial comparing embryo culture in two incubator systems: G185 KSystem versus EmbryoScope, Fertil Steril. 109 (2018) 302-9.e1. https://doi.org/10.1016/j.fertnstert.2017.10.008.

13. R. Sciorio, J.K. Thong, S.J. Pickering, Comparison of the development of human embryos cultured in either an EmbryoScope or benchtop incubator, J Assist Reprod Genet. 35 (2018) 515-22. https://doi.org/10.1007/s10815-017-1100-6.

14. C. Albert, N. Gonzalez, J. Marcos, L. Alegre, B.A. Ruiz, J.M. De Los Santos, M. Meseguer, P-028-The effect of high humidity culture conditions over embryo development: a continuous embryo monitoring assessment, Reproductive BioMedicine Online. 37 (2018) e15-e6. https://doi.org/https://doi.org/10.1016/j.rbmo.2018.04.029.

15. J.E. Swain, C. Graham, R. Kile, W.B. Schoolcraft, R.L. Krisher, Media evaporation in a dry culture incubator; effect of dish, drop size and oil on media osmolality, Fertility and Sterility. 110 (2018) e363-e4

16. Y. Boumerdassi, S. Huet, M. Millin, S. Sarandi, B. Bennani Smires, C. Sifer, [Impact of the type of incubator (non-humidified versus humidified) on embryo culture media osmolality], Gynecol Obstet Fertil Senol. (2020) S2468-7189(20)30351-2. https://doi.org/10.1016/j.gofs.2020.12.005.

17. C. Mori, M. Kuwayama, S.J. Silber, N. Kagawa, Y. Takehara, O. Kato, Water evaporation and osmolarity change of human embryo culture media in humid or in dry culture systems, Fertility \& Sterility. 94 (2010) S151-S

18. L.J. Van Winkle, N. Haghighat, A.L. Campione, Glycine protects preimplantation mouse conceptuses from a detrimental effect on development of the inorganic ions in oviductal fluid, J Exp Zool. 253 (1990) 215-9. https://doi.org/10.1002/jez.1402530211.

19. A.P. Tartia, N. Rudraraju, T. Richards, M.A. Hammer, P. Talbot, J.M. Baltz, Cell volume regulation is initiated in mouse oocytes after ovulation, Development. 136 (2009) 2247-54. https://doi.org/10.1242/dev.036756. 
20. J.M. Baltz, A.P. Tartia, Cell volume regulation in oocytes and early embryos: connecting physiology to successful culture media, Hum Reprod Update. 16 (2010) 166-76. https://doi.org/10.1093/humupd/dmp045.

\section{Tables}

\begin{tabular}{|c|c|c|c|c|}
\hline Index & DC mode & HC mode & $t$ or $x^{2}$ & $p$ value \\
\hline Oocyte number & 262 & 263 & & \\
\hline Fertility rate, $\%$ & 69.8 & 68.8 & 0.065 & 0.799 \\
\hline Cleavage rate, $\%$ & 98.4 & 98.9 & 0.176 & 0.675 \\
\hline High-quality embryo rate, \% & 66.1 & 55.3 & 4.392 & 0.036 \\
\hline
\end{tabular}




\begin{tabular}{|c|c|c|c|c|}
\hline Index & DC mode & $\mathrm{HC}$ mode & $\operatorname{tor} x^{2}$ & $p$ value \\
\hline Cycle & 184 & 136 & & \\
\hline Female age & $31.1 \pm 3.8$ & $31.5 \pm 4.1$ & 0.94 & 0.348 \\
\hline Male age & $33.3 \pm 5.1$ & $33.7 \pm 5.4$ & 0.756 & 0.450 \\
\hline Infertility time, year & $3.5 \pm 2.5$ & $3.4 \pm 2.4$ & 0.487 & 0.626 \\
\hline Primary infertility or secondary infertility & $93 / 91$ & $70 / 66$ & 0.027 & 0.910 \\
\hline BMI & $21.2 \pm 2.6$ & $21.7 \pm 2.7$ & 1.606 & 0.109 \\
\hline Cause of infertility & & & & 0.258 \\
\hline Factors from the female & 92 & 73 & & \\
\hline Factors from the male & 30 & 21 & 4.03 & \\
\hline Factors from the couples & 49 & 26 & & \\
\hline Unknown & 13 & 16 & & \\
\hline \multicolumn{5}{|l|}{ Ovarian stimulation regime } \\
\hline Long protocol & 32 & 40 & & \\
\hline Antagonist protocol & 95 & 58 & 7.462 & 0.059 \\
\hline Minimal stimulation protocol & 37 & 21 & & \\
\hline Others & 20 & 17 & & \\
\hline No. of oocytes retrieval & $9.6 \pm 6.3$ & $10.3 \pm 7.2$ & 0.851 & 0.396 \\
\hline IVF/ICSI & $128 / 56$ & $107 / 29$ & 3.328 & 0.074 \\
\hline High-quality embryo rate, \% & 58.2 & 53.0 & 5.57 & 0.018 \\
\hline \multicolumn{5}{|l|}{ Endometrial preparation protocol } \\
\hline Natural cycle & 82 & 57 & & \\
\hline Hormone replacement therapy & 95 & 74 & 0.245 & 0.885 \\
\hline Ovarian stimulation protocol & 7 & 5 & & \\
\hline No. of embryo transfer & $1.73 \pm 0.44$ & $1.87 \pm 0.34$ & 2.942 & 0.004 \\
\hline Clinical pregnancy rate, $\%$ & 64.7 & 58.1 & 1.438 & 0.246 \\
\hline Implantation rate, \% & 49.8 & 40.6 & 4.42 & 0.027 \\
\hline Abortion rate, $\%$ & 13.4 & 12.7 & 0.026 & 1.000 \\
\hline
\end{tabular}


Table 3 Offspring conditions obtained from dry culture and humid culture

\begin{tabular}{|lllll|}
\hline Index & DC mode & HC mode & t or $\mathbf{x}^{2}$ & $p$ value \\
\hline Singleton pregnancy & 68 & 49 & & \\
\hline Gestational age, w & $38.7 \pm 1.4$ & $39.1 \pm 1.4$ & 1.333 & 0.185 \\
\hline Preterm birth rate, \% & 10.3 & 6.1 & 0.634 & 0.517 \\
\hline Body weight, g & $3251 \pm 498$ & $3328 \pm 439$ & 0.862 & 0.391 \\
\hline Low birth weight rate, \% & 5.9 & 6.1 & 0.003 & 1.000 \\
\hline Anomaly rate, \% & 0 & 0 & & \\
\hline Twin pregnancy & 32 & 19 & & \\
\hline Gestational age, w & $35.9 \pm 2.2$ & $36.3 \pm 1.6$ & 0.799 & 0.428 \\
\hline Preterm birth rate, \% & 59.4 & 42.1 & 1.427 & 0.261 \\
\hline Body weight, g & $2428 \pm 746$ & $2534 \pm 451$ & 0.791 & 0.431 \\
\hline Low birth weight rate, \% & 51.6 & 44.7 & 0.445 & 0.544 \\
\hline Anomaly rate, \% & 1.6 & 2.6 & 0.142 & 1.000 \\
\hline
\end{tabular}

